\title{
MINIREVIEW
}

\section{The role of complex genomic alterations in neuroblastoma risk estimation}

\author{
Matthias Fischer* and Frank Berthold
}

\begin{abstract}
Specific genomic alterations, such as loss of the chromosomal region $11 \mathrm{q}$ or amplification of the oncogene MYCN, are well established markers of poor outcome in neuroblastoma. The advent of microarraybased comparative genomic hybridization (array-CGH) has enabled the analysis of pangenomic alteration profiles in the cancer genome, offering the possibility of identifying new prognostic markers from complex aberration patterns. Results from recent studies examining large primary neuroblastoma cohorts by array-CGH show that global genomic profiles may add significant prognostic information. Here, we discuss potential implications for risk estimation of neuroblastoma patients in clinical practice as well as for the understanding of neuroblastoma pathogenesis.
\end{abstract}

\section{The clinical heterogeneity of neuroblastoma}

Neuroblastoma, a pediatric malignancy of the developing sympathetic nervous system, is a multifaceted disease with biological and clinical courses ranging from relentless progression to spontaneous regression or differentiation into benign ganglioneuroma. Given these different phenotypes, therapeutic regimens vary between waitand-see approaches to the most intense multimodal treatment. Accurate prediction of the natural clinical course of each individual patient at the time of diagnosis is therefore an essential prerequisite for therapeutic decision-making. Clinical variables such as stage of the disease and age of the patient at diagnosis are well established predictors of neuroblastoma outcome. In addition, non-random cytogenetic aberrations have been shown to be associated with clinical courses in neuroblastoma and are increasingly used in risk stratification systems

*Correspondence: matthias.fischer@uk-koeln.de

Department of Pediatric Oncology and Hematology, University Children's Hospital, and Center for Molecular Medicine Cologne (CMMC), Kerpener Str. 62, 50924 Cologne, Germany (reviewed in [1-3]). Whereas amplification of the oncogene $M Y C N$ and several other genomic alterations, such as loss of the chromosomal regions $1 \mathrm{p}$ and 11q or gain of 17q, have been shown to be strong markers of poor outcome, hyper-diploidy of the tumor cells is associated with a favorable clinical phenotype [4]. However, whereas current risk estimation systems for neuroblastoma mostly succeed in discriminating patients with divergent outcomes, further improvements are required to prevent fatal events in low-risk and intermediate-risk groups and to avoid unnecessary cytotoxic treatment of patients in whom spontaneous regression will occur.

\section{Clinical significance of complex chromosomal alterations in neuroblastoma}

The advent of microarray-based comparative genomic hybridization (array-CGH) has facilitated the analysis of chromosomal alterations in the cancer genome, providing pangenomic alteration profiles with exceptional spatial resolution in a single experiment [5]. Initial arrayCGH studies of primary neuroblastomas [6,7] confirmed the clinical significance of known copy number variations and narrowed down breakpoint regions of non-random chromosome aberrations. In a recent survey, Caren et al. [8] investigated 165 primary neuroblastomas using Affymetrix $250 \mathrm{~K}$ single nucleotide polymorphism arrays and compared the survival of patient subgroups defined by genomic alterations. Patients with only numerical chromosomal aberrations and no other alteration had a favorable long-term outcome. In contrast, the survival of patients characterized by $M Y C N$ amplification, loss of 11q or gain of $17 \mathrm{q}$ was considerably worse, whereas no death or disease was observed in patients with tumors harboring segmental chromosome alterations other than those previously mentioned. These findings support results from previous studies indicating that a limited number of predictive genomic alterations are sufficient for risk assessment of neuroblastoma patients (reviewed in [2]).

Results from another recent survey by JanoueixLerosey et al. [9], however, indicated that global genomic profiles may add significant prognostic information to current neuroblastoma risk estimation. In this study [9], the prognostic significance of overall genomic alterations 
was investigated in a cohort of 493 primary neuroblastomas by bacterial artificial chromosome array-CGH. Whereas patients with tumors showing only numerical chromosome aberrations had an excellent survival, those with tumors harboring segmental genomic alterations showed a high risk of relapse and a poor outcome. Amplification of $M Y C N$ was confirmed to be a strong predictor of adverse outcome, but other single genomic alterations, such as loss of $11 \mathrm{q}$ or gain of $17 \mathrm{q}$, were overridden by the presence of any kind of segmental alterations in multivariate analyses.

Another significant difference between these two studies $[8,9]$ was noticed in the fraction of tumors with only numerical chromosome alterations. In the work of Janoueix-Lerosey et al. [9], this subgroup comprised $47 \%$ of the tumors, whereas it accounted for $28 \%$ of the cases in the study of Caren et al. [8]. Similar to the latter findings [8], this subgroup constituted $21 \%$ of the cases in a preliminary analysis of our array-CGH data [3]. These differences might in part be attributed to distinct compositions of the cohorts under investigation. However, they may also result from the lower spatial resolution of the microarrays used in the study of Janoueix-Lerosey et al. [9] than in the other surveys [3,8], which might have resulted in the detection of a smaller fraction of tumors with small gains or deletions and in the classification of fewer patients into subgroups with segmental aberrations. Taken together, although the results of these two comprehensive studies [8,9] are promising with respect to prognostic classification of neuroblastoma using array- $\mathrm{CGH}$, the clinical significance of global genomic alterations needs to be further evaluated in independent studies and compared with current risk estimation strategies.

An inherent disadvantage of array-CGH analysis is its propensity to disregard low-level copy number losses or gains in samples with a high proportion of contaminating stromal cells. This potential bias has been taken into account by Janoueix-Lerosey et al. [9] by analyzing only samples with a tumor content of at least $60 \%$, whereas the tumor content was not specified in the study of Caren et al. [8]. This discrepancy in the experimental set-up may have resulted in a higher fraction of flat genomic profiles (that is, with no alterations) in the latter study (19\%) [8] as compared with the former study (4\%) [9]. This suggestion is supported by the finding of only $2 \%$ flat genomic profiles in another study in which a tumor content of $60 \%$ had been used for sample selection [6]. Because of the rare occurrence of neuroblastomas without any chromosomal alterations, the clinical outcome of these patients has so far remained elusive. Nevertheless, the routine application of array-CGH in clinical practice might be considerably limited by the issue of contaminating stromal cells, because defined thresholds of tumor content will a priori exclude a substantial fraction of samples from the analysis. In addition, genomic heterogeneity within a single tumor might be missed by array$\mathrm{CGH}$ analysis. Although the frequency and the clinical consequences of genomic heterogeneity in neuroblastoma need to be clarified [10], it might be advisable to complement array-CGH analyses of neuroblastoma samples with methods for detecting chromosomal aberrations on the single cell level, such as fluorescence in situ hybridization, to evaluate the concordance of the results and to validate the clinical implications in large patient cohorts.

As an alternative to the overall genomic pattern as a prognostic marker, several reports have provided compelling evidence that specific gene-expression patterns can predict the natural courses of neuroblastoma patients with unprecedented accuracy [11-15]. These studies have shown that gene-expression-based classifiers can distinguish patients with contrasting clinical courses in almost all prognostic subgroups, including those defined by prognostic genomic makers such as $M Y C N$ amplification or loss of $11 \mathrm{q}[11,14]$. A systematic comparison of global genomic and transcriptomic classification results is still lacking, however. The routine application of expressionbased prognostic markers in clinical practice might be limited by the instability of mRNA in comparison with DNA, which will require strict adherence to elaborated standard operating procedures in the processing of tumor samples. In addition, similar to array-CGH approaches, classification results of gene-expressionbased predictors might be influenced by the relative amounts of stromal cells in the samples. In contrast to classifications based on genomic alterations, however, the prognostic significance of gene-expression profiles might be conferred by the stromal cells themselves, as has been described in other cancer entities, such as lymphoma or breast cancer $[16,17]$. Re-evaluation of the gene functions from existing gene-expression classifiers and validation of the predictive accuracy in neuroblastoma cohorts with low tumor contents will reveal the contribution of nontumorous cells to the prognostic validity of geneexpression-based classifiers in neuroblastoma.

\section{Biological classification of neuroblastoma by chromosome alterations}

Because of the strong association of numerical and segmental cytogenetic alterations with patient outcome, it has been suggested that neuroblastoma comprises two distinct clinico-genetic classes [18]. The first type corresponds to patients with favorable outcome and is characterized by mitotic dysfunction leading to whole chromosome gains or losses, whereas the second type corresponds to aggressive disease and is characterized by defects in maintaining genomic stability leading to 
segmental chromosome alterations. This view is supported by the study of Janoueix-Lerosey et al. [9] Given the prevalence of MYCN amplification and loss of $11 \mathrm{q}$ in unfavorable neuroblastoma, and the inverse correlation between these aberrations in high-risk neuroblastoma, it has been furthermore hypothesized that the natural behavior of high-risk tumors is mainly conferred by these two aberrations $[19,20]$. In the work of Caren et al. [8], this suggestion was substantiated by the finding that patients with $M Y C N$ amplification and those with loss of 11q differed significantly in both their age at diagnosis and their median survival time. However, whereas the influence of $M Y C N$ amplification on aggressive growth in neuroblastoma has been mostly proven [1], the effect of 11q loss on neuroblastoma biology is less clear. In a recent integrative genomics analysis of primary neuroblastoma, it was demonstrated that tumors with loss of 11q make up two distinct biological subgroups that differ in their clinical phenotype as well as in their gene-expression patterns [11]. These results suggest that 11q loss is not a primary determinant of neuroblastoma tumor behavior, indicating that the biology of neuroblastoma is more complex than the association of genomic alterations with patient outcome might suggest. We expect that the emerging application of nextgeneration sequencing will unravel novel genomic alterations that contribute to the programming of the various neuroblastoma phenotypes, which will lead to a refined molecular classification of this malignancy.

\section{The future: will genomic profiles have prognostic value in the clinic?}

The prognostic significance of specific single genomic markers is well established in neuroblastoma, and has led to their implementation in current risk assessment. Recent studies suggested that overall genomic profiles may further improve neuroblastoma risk estimation. Before routine use in clinical practice, the prognostic impact of global genomic alterations needs to be validated prospectively and compared with current stratification systems. In addition, it needs to be evaluated whether analysis of overall genomic profiles, geneexpression-based classifiers, or the combination of both will contribute most to an improved risk estimation of children with neuroblastoma. In any case, such analysis will require elaborate standard operating procedures to avoid technical pitfalls and defined interpretation guidelines to ensure reliable treatment stratification of each individual patient in future clinical trials.

\section{Abbreviations}

Array-CGH, microarray-based comparative genomic hybridization.

Competing interests

The authors declare that they have no competing interests.
Authors' contributions

$M F$ and FB drafted the manuscript and gave approval of the final version.

\section{Acknowledgements}

This work was supported by grants from the Bundesministerium für Bildung und Forschung (BMBF) through the National Genome Research Network plus (NGFNplus, grant 01GS0895) and the Fördergesellschaft Kinderkrebs-

Neuroblastom-Forschung e.V.

Published: 19 May 2010

\section{References}

1. Schwab M, Westermann F, Hero B, Berthold F: Neuroblastoma: biology and molecular and chromosomal pathology. Lancet Oncol 2003, 4:472-480.

2. Fischer M, Spitz R, Oberthur A, Westermann F, Berthold F: Risk estimation of neuroblastoma patients using molecular markers. Klin Padiatr 2008, 220:137-146.

3. Oberthuer A, Theissen J, Westermann F, Hero B, Fischer M: Molecular characterization and classification of neuroblastoma. Future Oncol 2009, 5:625-639.

4. Cohn SL, Pearson AD, London WB, Monclair T, Ambros PF, Brodeur GM, Faldum A, Hero B, lehara T, Machin D, Mosseri V, Simon T, Garaventa A, Castel V, Matthay KK: The International Neuroblastoma Risk Group (INRG) classification system: an INRG Task Force report. J Clin Oncol 2009, 27:289-297.

5. Pinkel D, Albertson DG: Array comparative genomic hybridization and its applications in cancer. Nat Genet 2005, 37 Suppl:S11-S17.

6. Spitz R, Oberthuer A, Zapatka M, Brors B, Hero B, Ernestus K, Oestreich J, Fischer M, Simon T, Berthold F: Oligonucleotide array-based comparative genomic hybridization (aCGH) of 90 neuroblastomas reveals aberration patterns closely associated with relapse pattern and outcome. Genes Chromosomes Cancer 2006, 45:1130-1142.

7. Stallings RL, Nair P, Maris JM, Catchpoole D, McDermott M, O'Meara A, Breatnach F: High-resolution analysis of chromosomal breakpoints and genomic instability identifies PTPRD as a candidate tumor suppressor gene in neuroblastoma. Cancer Res 2006, 66:3673-3680.

8. Caren H, Kryh H, Nethander M, Sjoberg RM, Trager C, Nilsson S, Abrahamsson J, Kogner P, Martinsson T: High-risk neuroblastoma tumors with 11q-deletion display a poor prognostic, chromosome instability phenotype with later onset. Proc Natl Acad Sci USA, 107:4323-4328.

9. Janoueix-Lerosey I, Schleiermacher G, Michels E, Mosseri V, Ribeiro A, Lequin D, Vermeulen J, Couturier J, Peuchmaur M, Valent A, Plantaz D, Rubie H, Valteau-Couanet D, Thomas C, Combaret V, Rousseau R, Eggert A, Michon J, Speleman F, Delattre O: Overall genomic pattern is a predictor of outcome in neuroblastoma. J Clin Oncol 2009, 27:1026-1033.

10. Theissen J, Boensch M, Spitz R, Betts D, Stegmaier S, Christiansen H, Niggli F, Schilling F, Schwab M, Simon T, Westermann F, Berthold F, Hero B: Heterogeneity of the MYCN oncogene in neuroblastoma. Clin Cancer Res 2009, 15:2085-2090.

11. Fischer M, Bauer T, Oberthur A, Hero B, Theissen J, Ehrich M, Spitz R, Eils R, Westermann F, Brors B, Konig R, Berthold F: Integrated genomic profiling identifies two distinct molecular subtypes with divergent outcome in neuroblastoma with loss of chromosome 11q. Oncogene, 29:865-875.

12. Oberthuer A, Berthold F, Warnat P, Hero B, Kahlert Y, Spitz R, Ernestus K, Konig R, Haas S, Eils R, Schwab M, Brors B, Westermann F, Fischer M: Customized oligonucleotide microarray gene expression-based classification of neuroblastoma patients outperforms current clinical risk stratification. J Clin Oncol 2006, 24:5070-5078.

13. Tomioka N, Oba S, Ohira M, Misra A, Fridlyand J, Ishii S, Nakamura Y, Isogai E, Hirata T, Yoshida Y, Todo S, Kaneko Y, Albertson DG, Pinkel D, Feuerstein BG, Nakagawara A: Novel risk stratification of patients with neuroblastoma by genomic signature, which is independent of molecular signature. Oncogene 2008, 27:441-449.

14. Vermeulen J, De Preter K, Naranjo A, Vercruysse L, Van Roy N, Hellemans J, Swerts K, Bravo S, Scaruffi P, Tonini GP, De Bernardi B, Noguera R, Piqueras M, Canete A, Castel V, Janoueix-Lerosey I, Delattre O, Schleiermacher G, Michon J, Combaret V, Fischer M, Oberthuer A, Ambros PF, Beiske K, Benard J, Marques B, Rubie H, Kohler J, Potschger U, Ladenstein R, et al:: Predicting outcomes for children with neuroblastoma using a multigene-expression signature: a retrospective SIOPEN/COG/GPOH study. Lancet Oncol 2009, 10:663-671. 
15. Ohira M, Oba S, Nakamura Y, Isogai E, Kaneko S, Nakagawa A, Hirata T, Kubo H, Goto T, Yamada S, Yoshida Y, Fuchioka M, Ishii S, Nakagawara A: Expression profiling using a tumor-specific cDNA microarray predicts the prognosis of intermediate risk neuroblastomas. Cancer Cell 2005, 7:337-350.

16. Finak G, Bertos N, Pepin F, Sadekova S, Souleimanova M, Zhao H, Chen H, Omeroglu G, Meterissian S, Omeroglu A, Hallett M, Park M: Stromal gene expression predicts clinical outcome in breast cancer. Nat Med 2008, 14:518-527.

17. Lenz G, Wright G, Dave SS, Xiao W, Powell J, Zhao H, Xu W, Tan B, Goldschmidt N, lqbal J, Vose J, Bast M, Fu K, Weisenburger DD, Greiner TC, Armitage JO, Kyle A, May L, Gascoyne RD, Connors JM, Troen G, Holte H, Kvaloy S, Dierickx D, Verhoef G, Delabie J, Smeland EB, Jares P, Martinez A, Lopez-Guillermo A, et al: Stromal gene signatures in large-B-cell lymphomas. N Engl J Med 2008, 359:2313-2323.

18. Brodeur GM: Neuroblastoma: biological insights into a clinical enigma. Nat Rev Cancer 2003, 3:203-216.
19. Bilke S, Chen QR, Westerman F, Schwab M, Catchpoole D, Khan J: Inferring a tumor progression model for neuroblastoma from genomic data. J Clin Oncol 2005, 23:7322-7331.

20. Wang Q, Diskin S, Rappaport E, Attiyeh E, Mosse Y, Shue D, Seiser E, Jagannathan J, Shusterman S, Bansal M, Khazi D, Winter C, Okawa E, Grant G, Cnaan A, Zhao H, Cheung NK, Gerald W, London W, Matthay KK, Brodeur GM, Maris JM: Integrative genomics identifies distinct molecular classes of neuroblastoma and shows that multiple genes are targeted by regional alterations in DNA copy number. Cancer Res 2006, 66:6050-6062

doi:10.1186/gm152

Cite this article as: Fischer $\mathrm{M}$, Berthold F: The role of complex genomic alterations in neuroblastoma risk estimation. Genome Medicine 2010, 2:31. 